\title{
The scope of no return: Openness predicts the spatial distribution of Inhibition of Return
}

\author{
Kristin E. Wilson ${ }^{1} \cdot$ Matthew X. Lowe $^{1} \cdot$ Justin Ruppel $^{1}$ • \\ Jay Pratt $^{1} \cdot$ Susanne Ferber ${ }^{1,2}$
}

Published online: 20 October 2015

(C) The Psychonomic Society, Inc. 2015

\begin{abstract}
How and what we attend to is foundational in determining the content of our experience, thus differences in attention contribute significantly to how we perceive the world, learn, and develop. Personality also plays a role in constraining how we learn to perceive the world and it is conceivable that some facets of personality interact with visual attention; however, the relationship between these two constitutional aspects of psychology remains unclear. To address this interplay between cognition and personality, we looked at how the Big Five personality traits relate to the spatial scope of attention, as indexed by the spatial distribution of Inhibition of Return (IOR). IOR is marked by a decrement in reaction time when a target appears at a cued location, more than $200 \mathrm{~ms}$ after that cue. As the cue/target distance increases there is a release from inhibition, providing a measure of the spatial distribution of IOR and reflecting the spatial scope of attention. The results presented here show personality does predict the distribution of IOR. Specifically, higher trait Openness is associated with a broader distribution of IOR and attention. This finding suggests there is an intimate connection between personality, particularly Openness, and the spatial allocation of attention.
\end{abstract}

Keywords Visual attention - Inhibition of return ·

Personality $\cdot$ Individual differences $\cdot$ Cognitive style

Kristin E. Wilson

kristin.wilson@utoronto.ca

1 Department of Psychology, University of Toronto, 100 St. George Street, Room 4020, Toronto, Ontario M5S 3G3, Canada

2 Rotman Research Institute, Baycrest, Toronto, Ontario, Canada

\section{Introduction}

Attention is a keystone cognitive function, yet it is largely defined by its effect on other cognitive processes - specifically, the way in which directing attention to something enhances processing, which involves selection of that which is attended and suppression of that which is not (Desimone \& Duncan, 1995; James, 1890; Kastner, De Weerd, Desimone, \& Ungerleider, 1998). This finely tuned filtering mechanism reduces the bandwidth of information the visual system has to process, which enables healthy and efficient cognitive functioning and plays an important role in determining the contents of conscious and unconscious experience and memory (Chun \& Turk-Browne, 2007). For example, individual differences in the ability to attend to relevant and ignore irrelevant information predict visual short-term memory (VSTM) capacity (McNab \& Klingberg, 2007; Vogel, McCollough, \& Machizawa, 2005). If attention is withdrawn from a stimulus, however, perceptual performance suffers (Yeshurun \& Carrasco, 1998).

In addition to the different components of attention such as alerting, orienting, and shifting (Posner \& Boies, 1971), attention can be described by its selectivity and scope or spatial distribution. Selectivity and scope are likely interdependent, just as increasing the span of a light beam reduces the intensity of the light. Narrow and highly selective attention may be an advantage in some situations, such as when one is required to fixate on relevant information when solving a complex arithmetic problem (Wiley \& Jarosz, 2012) or during rule-based learning (DeCaro, Thomas, \& Beilock, 2008). Broadening the spatial scope of attention may spread attentional resources required for selectivity more diffusely, possibly reducing the selective power in a given area (Eriksen \& St. James, 1986). There are situations, however, in which broad or diffuse attention, paired with less selectivity, is more advantageous, for 
instance in some forms of search (Smilek, Enns, Eastwood, \& Merikle, 2006), information-integration learning (DeCaro et al., 2008), ensemble processing (Alvarez \& Oliva, 2009), and creative problem solving (Wiley \& Jarosz, 2012). The delicate balance between diffusely distributed and selective attention may be differentially tipped by the fluid nature of task characteristics, mood, or emotion (Schmitz, De Rosa, \& Anderson, 2009) or by stable personality traits: some individuals may show a stronger propensity to engage one attentional mode over another. For instance, temperament predicts individual differences in attention (Derryberry \& Reed, 1994, 2002; Rothbart, Ahadi, \& Evans, 2000; Rueda, Rothbart, McCandliss, Saccomanno, \& Posner, 2005).

How we attend alters experience, learning, and development, and consequently impacts who we become. Thus individual differences in how attention is deployed may explain why the Big Five personality traits are associated with various different cognitive outcomes, such as academic success and career choice (Ackerman, 1996; Judge, Higgins, Thoresen, \& Barrick, 1999). While research on attention and the Big Five personality traits has traditionally developed in parallel with little crossover, recently more studies exploring how these two constitutional aspects of psychology influence each other have been emerging (MacLean \& Arnell, 2010; Risko, Anderson, Lanthier, \& Kingstone, 2012; Wu, Bischof, Anderson, Jakobsen, \& Kingstone, 2014).

\section{Personality and cognition}

The Big Five personality taxonomy is a widely used model to describe human personality traits (Costa \& McCrae, 1992; Digman, 1990; Soldz \& Vaillant, 1999) which have been found to be predictive of academic success (Furnham, Chamorro-Premuzic, \& McDougall, 2002; O'Connor \& Paunonen, 2007), intelligence, and vocational choices (Ackerman, 1996; Judge et al., 1999). These relationships suggest that different personality traits may be associated with different cognitive attributes. The link to attention may be most evident in Openness and Conscientiousness. Conscientiousness is characterized by being planful and organized, responsible and careful, achievement-oriented and persistent, all attributes that likely rely on the strength of top-down control of attention. Some research shows a relationship between Conscientiousness and attention, as Conscientiousness is negatively associated with Attention Deficit Disorder (Nigg et al., 2002), and positively predictive of career, academic success, and performance on highly structured tasks (Ackerman, 1996; Furnham et al., 2002; Judge et al., 1999) that depend on the ability to maintain focused attention. Openness, on the other hand, is characterized by active imagination, exploration, novelty-seeking, creativity, and intellectual curiosity. McCrae (1994) suggests that Openness reflects a preference specifically for the new and the different, as these individuals show an openness to many aspects of experience, including emotion, sensory experience, and ideas. Indeed, Openness is associated with cognitive tasks that arguably tap into a more broad and flexible attentional style, such as divergent thinking and creative problem solving (McCrae, 1987). A broader or more flexible attentional scope might allow for more information to enter short-term or working memory, facilitating initial processing of broadly or rapidly presented information.

This attentional-personality hypothesis has been tested using an attentional blink paradigm, in which letters are presented in rapid sequence and participants are asked to press a key in response to various targets (MacLean \& Arnell, 2010). Participants often miss the detection of a second target (T2), the attentional blink (AB), when it is presented in close temporal proximity to a previous target (T1). This "blink" is suspected to result from the capacity-limited nature of attentional processing with the first target depleting attentional resources. There are, however, individual differences in the extent to which T1 depletes resources and T2 is missed (e.g., MacLean, Arnell, \& Busseri, 2010; Rokke, Arnell, Koch, \& Andrews, 2002). Interestingly, Openness is associated with a greater T2 accuracy and smaller AB magnitude (less temporal delay required to perceive $\mathrm{T} 2$ ), while Conscientiousness is associated with greater accuracy for $\mathrm{T} 1$ and larger $\mathrm{AB}$ magnitude (MacLean \& Arnell, 2010). These results suggest that individuals high in Openness distribute attention more flexibly and broadly, while those high in Conscientiousness may allocate their attention more narrowly and rigidly. The $\mathrm{AB}$ task probes the temporal dynamics of attention; however, the spatial distribution of attention may also correspond with personality. If Openness is indeed associated with a broader allocation of attention, then we would expect to see a decrease in the degree of selectivity or an increase in processing of peripheral information. Consistent with this prediction, Openness has been associated with increased processing of irrelevant information (Peterson, Smith, \& Carson, 2002).

We believe that the relationship between these two personality traits, Conscientiousness and Openness, and various cognitive outcomes is related to distinct attentional styles. We hypothesize that Conscientiousness is associated with a more narrow distribution of attention while Openness is associated with a broader scope of attention. Though there has been work showing relationships between the Big Five and indirect measures of attention (e.g., Peterson et al., 2002; Risko et al., 2012), special forms of attention, such as social attention (Wu et al., 2014), and the temporal dynamics of attention (MacLean \& Arnell, 2010), the relationship between individual differences in The Big Five personality traits and the spatial gradient of visual attention has not been directly explored. 


\section{Inhibition of return and attention}

Previous work in the attention literature has demonstrated a spatial gradient to attention (Eriksen \& St. James, 1986; Eriksen \& Yeh, 1985); however, these paradigms have used few and fairly discrete probe locations. Revealing individual differences in the distribution of attention requires the use of display configurations with higher spatial resolution. Further, it may take a little time for attention to reach its full spatial scope and thus we sought a paradigm that would provide enough time for attention to distribute around a cued location, as there is likely more variance in this type of paradigm that may be accounted for by individual differences in personality. At longer cue/target SOAs (greater than $200 \mathrm{~ms}$ ), however, the typical response time (RT) facilitation when the target appears at a cued location turns into a decrement in RT, a cognitive phenomenon known as Inhibition of Return (IOR). It is believed that IOR reflects a bias towards the selection of novel locations over previously attended ones, by temporarily inhibiting the return to already searched locations, which is thought to facilitate foraging (Klein \& MacInnes, 1999). Thus, IOR can be thought of as an attentional bias away from already attended locations, a process that is mediated by the storage of these locations in VSTM (Castel, Pratt, \& Craik, 2003). It has been shown that IOR is not restricted to the cued location, but spreads to some degree within the cued hemifield (Collie, Maruff, Yucel, Danckert, \& Currie, 2000). By systematically varying the distance between cue and target over many locations, Bennett and Pratt (2001) revealed a fairly linear spatial distribution of IOR (RTs decrease with distance from cue). As such, Bennett and Pratt's IOR task fit our criteria for exploring individual differences in the distribution of attention very well, as it enabled sufficiently high spatial resolution and included enough temporal lag between cue and target that we would expect to see some individual differences in the distribution of attention emerge. While the nature of the present experimental work was exploratory, our a priori hypotheses were specific to the two personality traits that correlate with cognitive outcomes most strongly, namely Conscientiousness and Openness. We hypothesized individuals high in Conscientiousness would show a more narrow distribution of attention and spread of IOR, while Open individuals would show a broader or more diffuse distribution of attention and spread of IOR.

\section{Methods \\ Participants}

Fifty-four University of Toronto students (40 female, age 1731 years) participated in the study for course credit or $\$ 20$. Eight participants were excluded from analyses due to non- compliance with the task instructions and aberrant responses during the task (see exclusions below). The remaining 46 participants were included in the analyses. All participants had normal or corrected-to-normal vision, no history of psychiatric disorders, and gave informed consent. Given the exploratory nature of this work our sample size was established prior to collecting data based on what has been used in previous studies exploring individual differences in personality and attention (MacLean \& Arnell, 2010; Risko et al., 2012; Wu et al., 2014).

\section{Procedure}

First, participants completed the 120-item IPIP-NEO (International Item Pool-Neuroticism, Extraversion and Openness) questionnaire, a well validated tool to describe personality traits (Goldberg et al., 2006). Following this, participants completed the IOR task. Participants were instructed to respond as quickly as possible to the target (solid square) by pressing the spacebar. They were also informed that an irrelevant cue would appear on the screen prior to the target on most trials, but this should be ignored, as it was not predictive of target location. Participants performed several minutes of practice on this task while the experimenter monitored their eye movements. Once participants could maintain fixation during the task (average of 3-5 min) the experimental session was initiated. Participants were monitored during the experimental session via a video camera.

\section{Inhibition of Return (IOR) task}

The IOR task consisted of an invisible $11 \times 11$ grid (121 locations), with a center-to-center distance between neighboring locations of $1^{\circ}$ of visual angle (Fig. 1). On each trial, a single irrelevant cue (square outline, $1 \times 1^{\circ}$ of visual angle)

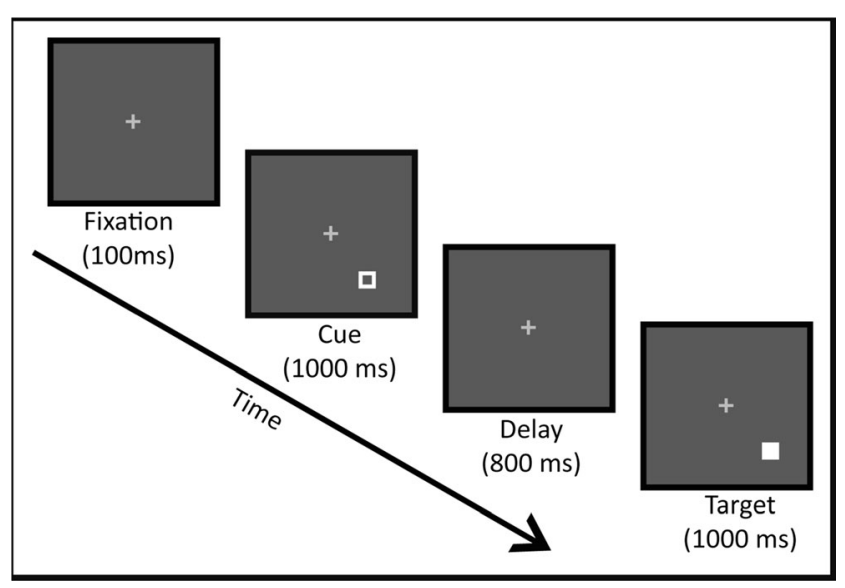

Fig. 1 Schematic representation of the Inhibition of Return (IOR) task. A cue appeared at one of four possible locations. After an 800-ms delay, a target appeared in one of 120 possible locations. Participants were to press a button as soon as they detected a target on the screen 
appeared for 1,000 ms at one of four possible locations, centered in one quadrant of the screen. After an $800-\mathrm{ms}$ delay, a single target (solid square, filling one location, $1 \times 1^{\circ}$ of visual angle) appeared for $1,000 \mathrm{~ms}$, at one of the 120 grid locations - targets never appeared at fixation (see Fig. 2a). Participants were instructed to maintain fixation on the cross at the center of the screen. The task consisted of 1,120 trials - 960 targetpresent trials (eight trials per location, 240 per quadrant) and 160 catch trails ( 40 per quadrant) where no target appeared to determine whether participants were attending to the task. The dependent variable was RTs.

\section{Exclusion}

Given that our primary research question concerned individual differences in attention, it is important that we exclude trials where participants were likely not attending or automatically responding. Specifically, one could presume that individuals high in Conscientiousness follow instructions to a greater degree than individuals who are low in Conscientiousness. Because there is no measure of accuracy on our task we trimmed the data using RT thresholds. Trials with RTs faster than $100 \mathrm{~ms}$ (reflecting random key presses) and slower than 1,500 ms were excluded from analyses (trialbased rejections), as these were taken to reflect inattention during a given trial. The majority of RTs fit well within this selection window (median $=310 \mathrm{~ms}$, standard deviation $(\mathrm{SD})=44)$. Eight participants were excluded from analyses altogether due to at least one of the following reasons, although most met more than one of these exclusion criteria: (1) Percentage of trial-based rejections exceed the mean rejection rate by at least $3 \mathrm{SDs}$; (2) The trials rejected included those required to calculate IOR (e.g., target appearing at the cued location), precluding our ability to measure IOR in the standard way in that participant; and (3) The percentage of catch trials a given participant responded to exceeded the

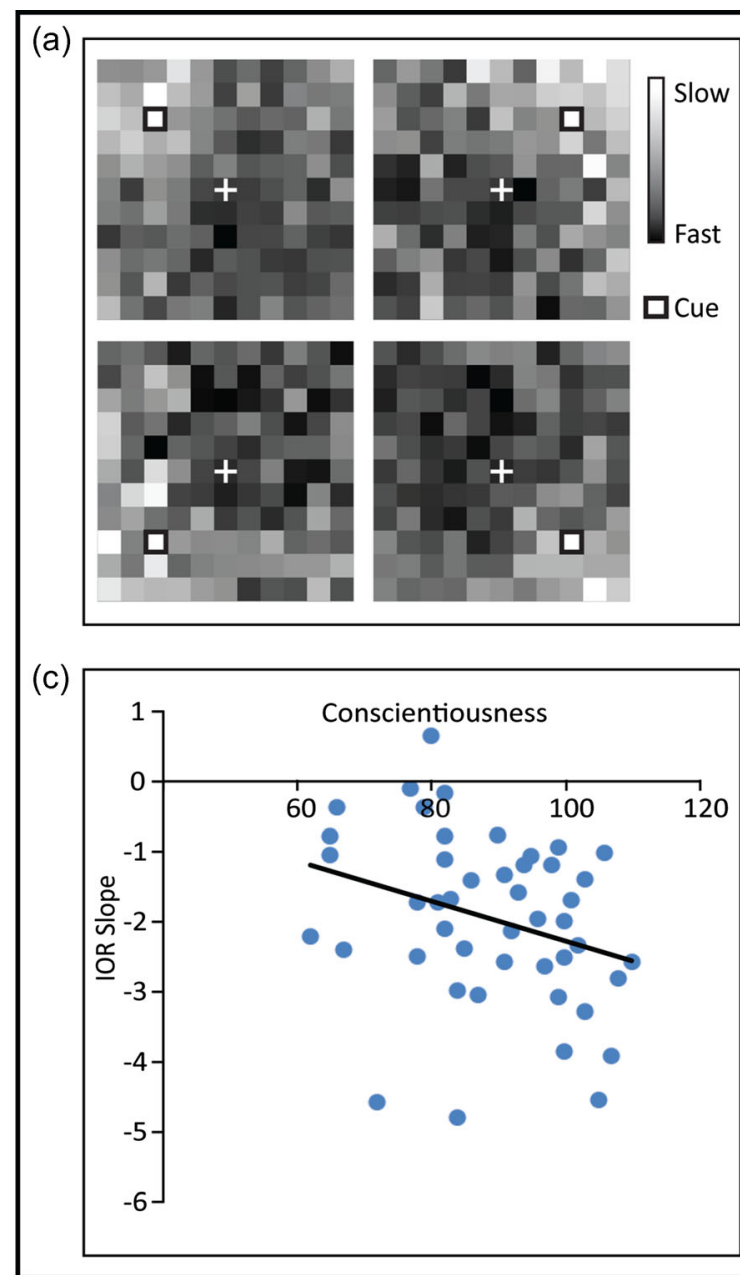

Fig. 2 Inhibition of Return (IOR) task results: (a) This reaction time (RT) plot shows the spatial distribution of IOR, with slower (lighter coloued) RTs for targets appearing close to cued locations and a gradual decrease in RTs with increasing distance, which is reflected in (b), the significant correlation between target/cue distance and RT. As target locations

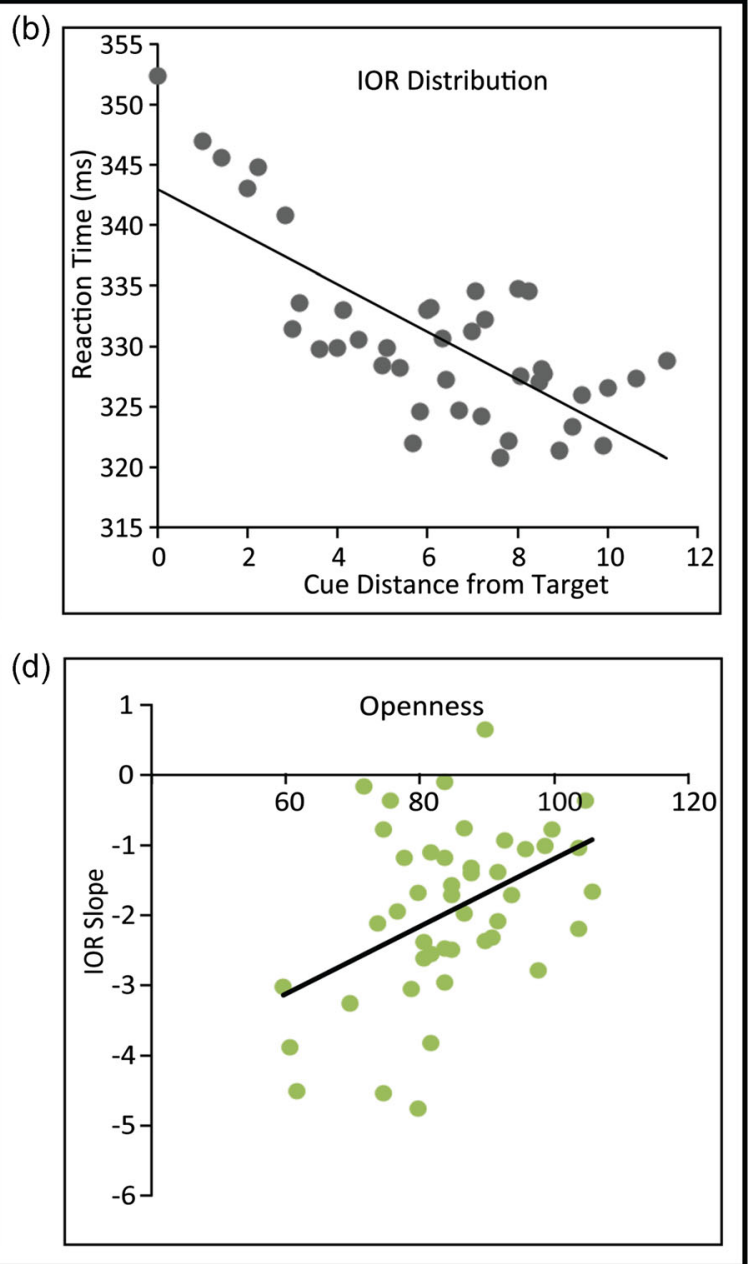

increase in distance from cued locations, RTs become faster. (c) Higher Conscientiousness predicted a larger negative slope, indicating a more narrow distribution of attention $(r=-.3, p=0.04)$; (d) conversely, higher Openness predicted a smaller (shallow) slope, indicating a more broad distribution of attention $(r=.44, p=0.002)$ 
mean by at least 3 SDs. These three measures were taken as indicators that a particular participant was likely not attending consistently throughout the task. Using Pearson's correlations, we checked to see whether personality was related to our exclusion criteria. There is no significant relationship with percentage of trials rejected and Conscientiousness $(r=0.00$, $p=0.99)$ or Openness $(r=.24, p=0.08)$. There is also no relationship with Conscientiousness and percentage of catch trials $(r=-.1, p=0.46)$, but there does appear to be some relationship with Openness and catch trials $(r=.39, p=$ 0.003). This relationship is driven by two extreme outliers, as it becomes non-significant after removal of these two participants $(r=.23, p=0.10)$. Overall, we did not find strong evidence in our data for a relationship between personality and exclusion criteria; however, this does not preclude the possibility of establishing such a relationship in future studies that are explicitly designed to explore how personality may interact with task instructions.

\section{Results}

\section{Individual differences in the scope of attention}

We observed a clear effect of IOR, such that RTs are slower when the target appears at cued locations $(M=359 \mathrm{~ms})$ relative to non-cued locations (center of adjacent and opposite quadrants, $M=336 \mathrm{~ms} ; t(45)=2.73, M$ difference $=22.43$, $p=0.009$ ) (descriptive statistics can be found in Table 1). Further, using linear regression analysis, we found a strong negative relation between distance and RTs across the whole group of 46 participants, such that RTs decrease as a function of distance from the cue $(R=-.74, b=-0.28, F(1,39)=45.9$, $p<0.001)-$ consistent with the Bennett and Pratt findings (Fig. 2a and b).

To explore the prediction that while Conscientiousness is associated with a more narrow scope of attention, Openness is associated with a broader scope of attention, we calculated regression equations of the spatial distribution of IOR for each individual, plotting RTs as a function of distance (as above).

Table 1 Descriptive statistics of personality variables and reaction times (RTs)

\begin{tabular}{lrl}
\hline Variable & Mean & SE \\
\hline Conscientiousness & 88.85 & 1.88 \\
Openness & 83.87 & 1.62 \\
RT at cued location & 358.85 & 9.31 \\
RT at uncued location & 336.42 & 7.82 \\
Average RT & 343.90 & 7.39 \\
Slope of IOR & -2.00 & 0.18 \\
\hline
\end{tabular}

SE standard error, IOR Inhibition of Return
We took the slope of this equation for each individual as an indication of the spatial gradient of IOR/attention. For instance, if someone had a steep or large negative slope this would indicate that they quickly recovered from IOR over cue/target distance, suggesting a narrow distribution of IOR/ attention and vice versa for individuals with a shallow or small negative slope. This measure isolates the rate of change across space within each individual, thus it is a relative measure of the distribution of attention. Examining the relationship between this measure of the scope of IOR or attention and personality using Pearson's correlations, we find both Conscientiousness and Openness correlate with the spatial gradient of IOR (Fig. 2c and b). Conscientiousness is associated with a steep (more negative) RT spatial gradient ( $r=-.3$, $\left.p=0.04, r^{2}=-.1\right)$, such that their RTs are finely tuned to cue/ target proximity; their narrow focus of attention results in a small area of inhibition around the cued location with an accelerated release from inhibition. Individuals high in Openness, on the other hand, show a shallow RT spatial gradient $\left(r=.44, p=0.002, r^{2}=.19\right)$; their spatially diffuse scope of attention leads to more widespread inhibition.

Not all individuals showed a strong IOR effect, thus it is possible that individuals with a weak IOR response may have impacted the observed relationship between personality and the distribution of IOR. Therefore, we ran separate analyses including only those individuals that showed clear IOR (RT slower for target at cued location relative to non-cued locations). The Pearson's correlations between Openness and IOR slope remained intact $(r=.49, p=0.004)$, while the relationship with Conscientiousness became marginal $(r=-.27, p=$ 0.12 ). Though not all participants showed the expected IOR effect, selective exclusion of these data points because they do not fit our hypotheses would be unwarranted. In the following analysis we report results with the full data set of 46 participants; however, the relationships reported here also held when analyses were restricted to just those showing a consistent IOR effect.

Though the Big 5 personality traits have canonically been thought to be independent, there is evidence of correlations between these factors, with Conscientiousness and Openness belonging to different latent variables (DeYoung, 2006; Digman, 1997). A five-predictor model with simultaneous entry (Neuroticism, Extroversion, Openness, Agreeableness, and Conscientiousness) was used to determine the unique amount of variance in IOR slope accounted for by each of the personality traits $(R=.54, F(5,40)=3.36, p=$ $0.013, \mathrm{R}^{2}=.30$ ) (see Table 2 for regression coefficients and partial correlations). Only Openness accounted for a significant portion of unique variance, over and above the other predictors $(r=.46, p r=.43, p=0.005)$.

It is possible that the relationship between RT on this task and personality may be driven by a third variable, such as a difference in processing speed and/or the ability to stay on task 
Table 2 Regression coefficients for the degree to which each of the Big 5 personality traits predict Inhibition of Return (IOR) slope

\begin{tabular}{lrrrrr}
\hline Personality factor & \multicolumn{1}{l}{$B$} & SE $B$ & $\beta$ & $\begin{array}{l}\text { Semi-partial } \\
\text { correlation }\end{array}$ & VIF \\
\hline Neuroticism & -0.004 & 0.013 & .042 & .047 & 1.14 \\
Extroversion & -0.006 & 0.017 & -.051 & -.056 & 1.18 \\
Openness & 0.050 & 0.017 & .455 & .426 & 1.32 \\
Agreeableness & -0.008 & 0.015 & -.280 & -.296 & 1.16 \\
Conscientiousness & -0.003 & 0.015 & -.119 & -.120 & 1.36 \\
\hline
\end{tabular}

$\mathrm{R}^{2}=.30$, the $\mathrm{b}$ for Openness was significant, $\mathrm{p}=0.005$. B is the unstandardized coefficient and $\beta$ is the standardized coefficient

(vigilance), rather than the distribution of attention across space. We explored this possibility from three angles. First, we examined the relationship between slope and overall average RTs and we found that they are almost orthogonal measures $(r=.1, p=.5)$. Second, we assessed processing speed, as a function of average RT, by running Pearson's correlations on average RT across all target locations as well as average RT on trials where the target appeared at the cued location (site of peak IOR effect). This approach failed to reveal a significant relationship between personality and average RT (Openness, $r=.08, p=0.61$; Conscientiousness, $r=-.19, p=0.23)$ and personality and RT at the cued location (Openness, $r=-.01$, $p=0.93$; Conscientiousness, $r=-.10, p=0.51$ ). Lastly, one can hypothesize that personality may modulate how participants respond over the course of a given experimental session, due to variance in one's ability to maintain focus. We binned our data into four quartiles and compared RTs in the first and last quartile of responses across the entire experiment. We observed that participants were responding faster towards the end (with an average decrease in RT from the first to the last quartile of $22 \mathrm{~ms}$ ). This change over time, however, did not significantly correlate with either Openness $(r=-.22, p=$ $0.14)$ or Conscientiousness $(r=.37, p=0.71)$. We also looked at changes in variability across the task, calculated as a difference in standard deviation between the first and second half of the task. However, this relationship also did not reach significance with neither Openness $(r=-.1, p=0.52)$ nor Conscientiousness $(r=-.09, p=0.57)$. As such, we failed to find a significant relationship between overall RT and changes in RTs over time and personality. A lack of significance, however, does not unequivocally rule out that such a relationship exists. It is possible that some of these small but non-significant relationships are real and would reach significance with a larger sample.

Overall, our findings suggest that Conscientiousness may be related to a more focused distribution of attention, however, in a weaker sense than Openness, where we see a strong and statistically significant case for the association with the spatial distribution of IOR and the spatial scope of attention, regardless of overall processing speed.

\section{Discussion}

In summary, we find evidence here suggesting stable personality traits, such as Openness and Conscientiousness, are associated with more transient cognitive faculties, such as the spatial scope of attention. The spatial distribution or scope of attention was measured using a modified version of a standard IOR paradigm (Fig. 1a) (Bennett \& Pratt, 2001), in which RTs slow when a target appears at a cued location more than $200 \mathrm{~ms}$ after the offset of the cue (Posner \& Cohen, 1984). Across an invisible grid of $11 \times 11$ possible locations (Fig. 2a), we find the expected negative correlation between proximity and RTs across the whole group of 46 participants, such that RTs decrease as a function of distance from the cue - consistent with Bennett and Pratt's (2001) findings (Fig. 2a and b). Next, examining the relationship with personality we find both Conscientiousness and Openness correlate with the spatial gradient in RTs - an index of the scope of attention (Fig. 2c and d). Individuals high in Openness show a shallow (less negative) RT spatial gradient, such that their RTs are more broadly tuned to cue/target proximity; their spatially diffuse scope of attention leads to more widespread inhibition. Individuals high in Conscientiousness, on the other hand, show a steeper (more negative) RT spatial gradient, such that their RTs are more finely tuned to cue/target proximity; their narrow focus of attention results in a small area of inhibition around the cued location with an accelerated release from inhibition. We take these findings to reflect individual differences in the spatial distribution of attention.

Given that individuals high in Conscientiousness may have a greater degree of control over their attention (Ackerman, 1996; Nigg et al., 2002) and individuals high in Openness show reduced inhibition and greater response to distractors (Peterson et al., 2002), an alternative interpretation of our data suggests the steeper slopes seen in Conscientious individuals and shallower slopes in Open individuals may reflect differences in the ability to ignore the irrelevant cue and selectively attend to the target, rather than the spatial diffusion of attention per se. The amount of attentional resources allocated to the cue may affect the spread of IOR around the cued location and, as such, our results may reflect differences in the selectivity of attention rather than the distribution of attention per se. Though we cannot rule out differences in attention to the cue as a factor affecting our results, we do not believe variability in attention to the cue accounts for these particular results. If differences in orienting towards the cue were tied to personality we would expect RT specifically at the cued location to vary with personality; however, the relationships were small and far from significant and did not likely have a strong influence over our results. Further, it is unclear how a weaker or stronger allocation of attention to the cue might affect the distribution of IOR and the spatial spread of attention. These two aspects of attention, selectivity and scope, can 
be somewhat orthogonal, but this is beyond the scope of these exploratory data. An interesting empirical question for future work would be to look more closely at the selectivity of attention and personality by manipulating selectivity experimentally and/or exploring this effect on a faster timescale, for instance in an attentional facilitation paradigm, with faster cue/target SOAs.

Though it is fairly well established that orienting attention to the cue is a key mechanism in IOR (Klein, Christie, \& Morris, 2005; Klein \& MacInnes, 1999; Posner \& Cohen, 1984; Yantis \& Jonides, 1984) there is still some debate in the literature about the relative influence of perceptual contributions to this phenomenon. Zhao and Heinke (2014) suggest that attention and perception both play a role in IOR, but at different stages. It is known that attention spreads within and around objects (Egly, Driver, \& Rafal, 1994) and that attention modulates perceptual processing in early visual perceptual regions (Kastner et al., 1998). Truly disentangling the effects of attention from those of perception would require a version of this IOR task performed in the absence of attention. Given the effect attention has on perception, we believe the distribution of IOR we observed in the present study is best explained by the spatial effect of attention on visual processing, rather than the spread of visual perception per se; however, this is an empirical question for further investigation.

Though IOR, RTs, and RT slopes are often related to each other, we did not find a significant relationship between RT and RT slopes here, nor did personality predict average RT or $\mathrm{RT}$ at the cued locations. The weak relationship between personality and these two RT measures suggest that differences in general processing speed do not easily explain the relationship between personality and relative change in RT over space. It would be remiss of us, however, if we did not point out that the lack of significance reported here does not mean there is zero influence. For instance, Conscientiousness shows a nonsignificant relationship with average RT $(r=-.19)$; however, we do not know whether this relationship's p-value is significantly different from that of the significant relationship between Conscientiousness and IOR Slope $(r=-.3)$. Though only one of these two correlations reaches the significance threshold, the difference between the significant and not significant result is not directly tested (Gelman \& Stern, 2006). We cannot make any strong claims about the null finding with average RT; however, we can say there is little support for the alternative interpretation, that differences in processing speed underlie the relationship between personality and relative change in IOR over space. Further, there is some ambiguity to the significant and non-significant Conscientiousness results. Conscientiousness is defined by a quality of dutifulness, self-control, and ability to follow through on tasks, thus one may expect that our measure of Conscientiousness (NEOIPIP) would predict adherence to the task instructions (e.g., number of catch trials responded to or trials rejected); however, we failed to find a significant relationship. Though this brings some peace of mind, suggesting that our rejection criteria did not result in biased rejection of individuals, this raises questions about how well this questionnaire measured Conscientiousness. Thus, the seemingly weaker relationships with Conscientiousness in the present article may speak to limitations in the use of questionnaires in measuring the underlying construct of Conscientiousness or a lack of power, rather than a true absence of a relationship. Thus the relationship between Conscientiousness and narrow attentional scope remains somewhat ambiguous, requiring further exploration. We are more confident in our significant results showing a clear relationship between Openness and a broadened spatial distribution of attention.

Personality traits are part of a complex continuum, characterized by behavior, cognition, and affect (Zillig, Hemenover, $\&$ Dienstbier, 2002). From a developmental perspective it is unclear whether attentional biases drive the development of personality or vice versa, as there is evidence of genetic components to both attention (Faraone et al., 2005; Fossella et al., 2002) and personality (Jang, Livesley, \& Vemon, 1996; Loehlin, McCrae, Costa, \& John, 1998). We may be born with a propensity towards a particular attentional, motivational, and affective style, which all contribute to our personality. Through experience, attention and personality may continue to develop, mutually constraining each other. For instance, the broad distribution of attention in Open individuals may be influenced by a novelty bias associated with this trait (De Fruyt, Van De Wiele, \& Van Heeringen, 2000). In fact, individual differences in novelty bias may motivate the tuning of the scope of attention on this IOR task, as Dodd, van der Stigchel, and Hollingworth (2009) show that IOR arises specifically in tasks with a novelty bias (e.g., visual search).

Combining personality and attention research can be useful for enhancing our understanding of both attention and personality. For instance, these individual differences may help explain some of the variance in attention paradigms, particularly those that include distractors in the periphery such as paradigms used in addressing the debate regarding the nature of attentional capture (e.g., Folk, Ester, \& Troemel, 2009; Yantis $\&$ Jonides, 1990) or those that look at the distribution of attention more directly (e.g., Eriksen \& St. James, 1986). Using personality as an individual difference measure provides a novel way of clustering attention data, revealing nuances to attention missed by whole-group analysis. Future work should further elucidate how personality relates to other aspects of attention, such as the degree of selectivity and other capacities closely related to attention, for example, VSTM (Cowan, 2001). This work may then inform our understanding of personality, revealing a cluster of attentional and cognitive traits and behavioral correlates of Openness and Conscientiousness. 
Acknowledgments This research was supported by grants awarded to Susanne Ferber from the Canadian Institute of Health Research, the National Science and Engineering Research Council of Canada, and the Early Researcher Awards Program of the Province of Ontario.

\section{References}

Ackerman, P. L. (1996). A theory of adult intellectual development: Process, personality, interests, and knowledge. Intelligence, 22(2), 227-257.

Alvarez, G. A., \& Oliva, A. (2009). Spatial ensemble statistics are efficient codes that can be represented with reduced attention. Proceedings of the National Academy of Sciences, 106(18), 7345-7350.

Bennett, P. J., \& Pratt, J. (2001). The spatial distribution of inhibition of return. Psychological Science, 12(1), 76-80.

Castel, A. D., Pratt, J., \& Craik, F. I. (2003). The role of spatial working memory in inhibition of return: Evidence from divided attention tasks. Perception \& Psychophysics, 65(6), 970-981.

Chun, M. M., \& Turk-Browne, N. B. (2007). Interactions between attention and memory. Current Opinion in Neurobiology, 17(2), 177-184.

Collie, A., Maruff, P., Yucel, M., Danckert, J., \& Currie, J. (2000). Spatiotemporal distribution of facilitation and inhibition of return arising from the reflexive orienting of covert attention. Journal of Experimental Psychology: Human Perception and Performance, 26(6), 1733.

Costa, P. T., \& McCrae, R. R. (1992). Four ways five factors are basic. Personality and Individual Differences, 13(6), 653-665.

Cowan, N. (2001). The magical number 4 in short-term memory: A reconsideration of mental storage capacity. The Behavioral and Brain Sciences, 24(1), 87-114. discussion 114-185.

De Fruyt, F., Van De Wiele, L., \& Van Heeringen, C. (2000). Cloninger's psychobiological model of temperament and character and the fivefactor model of personality. Personality and Individual Differences, 29(3), 441-452.

DeCaro, M. S., Thomas, R. D., \& Beilock, S. L. (2008). Individual differences in category learning: Sometimes less working memory capacity is better than more. Cognition, 107(1), 284-294.

Derryberry, D., \& Reed, M. A. (1994). Temperament and attention: Orienting toward and away from positive and negative signals. Journal of Personality and Social Psychology, 66(6), 1128.

Derryberry, D., \& Reed, M. A. (2002). Anxiety-related attentional biases and their regulation by attentional control. Journal of Abnormal Psychology, 111(2), 225.

Desimone, R., \& Duncan, J. (1995). Neural mechanisms of selective visual attention. Annual Review of Neuroscience, 18(1), 193-222.

DeYoung, C. G. (2006). Higher-order factors of the Big Five in a multiinformant sample. Journal of Personality and Social Psychology, 91(6), 1138.

Digman, J. M. (1990). Personality structure: Emergence of the five-factor model. Annual Review of Psychology, 41(1), 417-440.

Digman, J. M. (1997). Higher-order factors of the Big Five. Journal of Personality and Social Psychology, 73(6), 1246.

Dodd, M. D., Van der Stigchel, S., \& Hollingworth, A. (2009). Novelty is not always the best policy inhibition of return and facilitation of return as a function of visual task. Psychological Science, 20(3), 333-339.

Egly, R., Driver, J., \& Rafal, R. D. (1994). Shifting visual attention between objects and locations: Evidence from normal and parietal lesion subjects. Journal of Experimental Psychology. General, 123(2), 161-177.
Eriksen, C. W., \& St. James, J. D. (1986). Visual attention within and around the field of focal attention: A zoom lens model. Perception \& Psychophysics, 40(4), 225-240.

Eriksen, C., \& Yeh, Y. (1985). Allocation of attention in the visual field. Journal of Experimental Psychology: Human Perception and Performance, 11(5), 583-597.

Faraone, S. V., Perlis, R. H., Doyle, A. E., Smoller, J. W., Goralnick, J. J., Holmgren, M. A., \& Sklar, P. (2005). Molecular genetics of attention-deficit/hyperactivity disorder. Biological Psychiatry, 57(11), 1313-1323.

Folk, C. L., Ester, E. F., \& Troemel, K. (2009). How to keep attention from straying: Get engaged! Psychonomic Bulletin \& Review, 16(1), $127-132$.

Fossella, J., Sommer, T., Fan, J., Wu, Y., Swanson, J. M., Pfaff, D. W., \& Posner, M. I. (2002). Assessing the molecular genetics of attention networks. BMC Neuroscience, 3(1), 14.

Furnham, A., Chamorro-Premuzic, T., \& McDougall, F. (2002). Personality, cognitive ability, and beliefs about intelligence as predictors of academic performance. Learning and Individual Differences, 14(1), 47-64.

Gelman, A., \& Stern, H. (2006). The difference between "significant" and "not significant" is not itself statistically significant. The American Statistician, 60(4), 328-331.

Goldberg, L. R., Johnson, J. A., Eber, H. W., Hogan, R., Ashton, M. C., Cloninger, C. R., \& Gough, H. G. (2006). The international personality item pool and the future of public-domain personality measures. Journal of Research in Personality, 40(1), 84-96.

James, W. (1890). The principles of psychology. New York: Holt.

Jang, K. L., Livesley, W. J., \& Vemon, P. A. (1996). Heritability of the big five personality dimensions and their facets: A twin study. Journal of Personality, 64(3), 577-592.

Judge, T. A., Higgins, C. A., Thoresen, C. J., \& Barrick, M. R. (1999). The big five personality traits, general mental ability, and career success across the life span. Personnel Psychology, 52(3), 621-652.

Kastner, S., De Weerd, P., Desimone, R., \& Ungerleider, L. G. (1998). Mechanisms of directed attention in the human extrastriate cortex as revealed by functional MRI. Science, 282(5386), 108-111.

Klein, R. M., Christie, J., \& Morris, E. P. (2005). Vector averaging of inhibition of return. Psychonomic Bulletin \& Review, 12(2), 295-300.

Klein, R. M., \& MacInnes, W. J. (1999). Inhibition of return is a foraging facilitator in visual search. Psychological Science, 10(4), 346-352.

Loehlin, J. C., McCrae, R. R., Costa, P. T., Jr., \& John, O. P. (1998). Heritabilities of common and measure-specific components of the Big Five personality factors. Journal of Research in Personality, 32(4), 431-453.

MacLean, M. H., \& Arnell, K. M. (2010). Personality predicts temporal attention costs in the attentional blink paradigm. Psychonomic Bulletin \& Review, 17(4), 556-562.

MacLean, M. H., Arnell, K. M., \& Busseri, M. A. (2010). Dispositional affect predicts temporal attention costs in the attentional blink paradigm. Cognition \& Emotion, 24(8), 1431-1438.

McCrae, R. R. (1987). Creativity, divergent thinking, and openness to experience. Journal of Personality and Social Psychology, 52(6), 1258.

McNab, F., \& Klingberg, T. (2007). Prefrontal cortex and basal ganglia control access to working memory. Nature Neuroscience, 11(1), $103-107$.

Nigg, J. T., John, O. P., Blaskey, L. G., Huang-Pollock, C. L., Willicut, E. G., Hinshaw, S. P., \& Pennington, B. (2002). Big Five dimensions and ADHD symptoms: Links between personality traits and clinical symptoms. Journal of Personality and Social Psychology, 83(2), 451

O’Connor, M. C., \& Paunonen, S. V. (2007). Big Five personality predictors of post-secondary academic performance. Personality and Individual Differences, 43(5), 971-990. 
Peterson, J. B., Smith, K. W., \& Carson, S. (2002). Openness and extraversion are associated with reduced latent inhibition: Replication and commentary. Personality and Individual Differences, 33(7), $1137-1147$.

Posner, M. I., \& Boies, S. J. (1971). Components of attention. Psychological Review, 78(5), 391.

Posner, M. I., \& Cohen, Y. (1984). Components of visual orienting. Attention and Performance X: Control of Language Processes 32: $531-556$.

Risko, E. F., Anderson, N. C., Lanthier, S., \& Kingstone, A. (2012). Curious eyes: Individual differences in personality predict eye movement behavior in scene-viewing. Cognition, 122(1), 86-90.

Rokke, P. D., Arnell, K. M., Koch, M. D., \& Andrews, J. T. (2002). Dualtask attention deficits in dysphoric mood. Journal of Abnormal Psychology, 111(2), 370.

Rothbart, M. K., Ahadi, S. A., \& Evans, D. E. (2000). Temperament and personality: Origins and outcomes. Journal of Personality and Social Psychology, 78(1), 122.

Rueda, M. R., Rothbart, M. K., McCandliss, B. D., Saccomanno, L., \& Posner, M. I. (2005). Training, maturation, and genetic influences on the development of executive attention. Proceedings of the National Academy of Sciences of the United States of America, 102(41), 14931-14936.

Schmitz, T. W., De Rosa, E., \& Anderson, A. K. (2009). Opposing influences of affective state valence on visual cortical encoding. The Journal of Neuroscience, 29(22), 7199-7207.

Smilek, D., Enns, J. T., Eastwood, J. D., \& Merikle, P. M. (2006). Relax! Cognitive strategy influences visual search. Visual Cognition, 14(48), 543-564.
Soldz, S., \& Vaillant, G. E. (1999). The Big Five personality traits and the life course: A 45-year longitudinal study. Journal of Research in Personality, 33(2), 208-232.

Vogel, E., McCollough, A., \& Machizawa, M. (2005). Neural measures reveal individual differences in controlling access to working memory. Nature, 438(7067), 500-503.

Wiley, J., \& Jarosz, A. F. (2012). How working memory capacity affects problem solving. Psychology of Learning and Motivation-Advances in Research and Theory, 56, 185.

Wu, D. W.-L., Bischof, W. F., Anderson, N. C., Jakobsen, T., \& Kingstone, A. (2014). The influence of personality on social attention. Personality and Individual Differences, 60, 25-29.

Yantis, S., \& Jonides, J. (1984). Abrupt visual onsets and selective attention: Evidence from visual search. Journal of Experimental Psychology: Human Perception and Performance, 10(5), 601-621.

Yantis, S., \& Jonides, J. (1990). Abrupt visual onsets and selective attention: Voluntary versus automatic allocation. Journal of Experimental Psychology: Human Perception and Performance, 16(1), 121-134.

Yeshurun, Y., \& Carrasco, M. (1998). Attention improves or impairs visual performance by enhancing spatial resolution. Nature, 396(6706), 72-75.

Zillig, L. M. P., Hemenover, S. H., \& Dienstbier, R. A. (2002). What do we assess when we assess a Big 5 trait? A content analysis of the affective, behavioral, and cognitive processes represented in Big 5 personality inventories. Personality and Social Psychology Bulletin, 28(6), 847-858. 